\title{
Kinesthetic Elementary Mathematics - Creating Flow with Gesture Modality
}

\author{
Jussi Okkonen, Sumita Sharma, Roope Raisamo, and Markku Turunen \\ University of Tampere, Finland \\ \{jussi.okkonen, sumita.s.sharma, roope.raisamo, markku.turunen\}@uta.fi
}

\begin{abstract}
Educational games for young children have boomed with the growing abundance of easy to use interfaces, especially on smartphones and tablets. In addition, most major gaming consoles boast of multimodal interaction, including the more novel and immersive gesture-based or bodily interaction; a concept proved by masses of consumers including young children.

In this paper, we examine an elementary mathematics learning application that aims to promote a state of flow for children aged between 6-8 years. The application is run on a PC and uses the Microsoft Kinect sensor for motion tracking. It provides gamified approaches to teach the number system between 0-20. Our underlying hypothesis is that kinesthetic learning methods supported by bodily interaction provide leverage to different types of learners.

The paper describes the results of two sets $\left(n^{1}=23, n^{2}=44\right)$ of pilot tests for exercise application for $P C$ and Kinect. The tools utilized include a short and simplified survey for the children, and another survey and open-ended questionnaire for the teachers. Our key findings relate to the user experience of gesture-based interaction and show how the gesture modality promotes flow. Furthermore, we discuss our preliminary assessment on several learning related themes.
\end{abstract}

Keywords: learning games, gesture interaction, flow, immersion

\section{Introduction}

Research has shown that bodily interaction technologies, such as, those employing spatial gesture recognition with the Microsoft Kinect sensor, are a promising way forward for immersive and engaging gamified learning environment [1][2][3], [4][5]. SMALLabs [6] and EdGE (Educational Gaming Environments) research labs are dedicated to understanding and designing educational games based on kinesthetic learning experiences. There is also an online community, called KinectEDucation, focused on promoting Kinect-based application in the classroom. Thus, current trends in technologies afford bodily interaction with digital content, allowing for bodily representation of abstract concepts. Furthermore, games that are challenging and highly interactive promote a state of flow. They provide realizable challenges with an appropriate level of feedback and reward to be engaging and interesting for the player, essential conditions for flow [7] and [8]. Studies have also shown that kinesthetic learning applications allow even the more restless of students to be able concentrate for longer durations [9] and [10] and retain the learned material longer [2][11].

Several interdisciplinary studies have claimed that abstract mathematical concepts are rooted in our bodily experiences [12] and [13]. For example, most children start learning to count by using their fingers and can also understanding quantities, such as one hand has five fingers without necessarily counting to confirm [14]. This creates opportunities for combining the benefits of bodily interaction to learning of abstract mathematical content, providing an immersive and engaging learning environment and creating a state of flow. However, such studies are limited to a few independent examples [4][15][16] and [17] and further research is required to understand the relation between bodily interaction and the state of flow. 
Our focus is on understanding the immersion, engagement and state of flow associated with bodily interaction based mathematical learning environments. Our kinesthetic elementary mathematics application is based on bodily interaction with digital content within quantity and number scale of 0 to 20. It extends the idea of a typical digital learning application towards more traditional physical setting by allowing children to use their body movement in the interaction. Moreover, the underlying assumption is that different types of learner benefit from bodily interaction as it increases immersion and engagement thus building flow. Using our application, we conducted two user studies in the classroom environment with children between 6-8 years $(n=67)$. We used tools developed by Shernoff, Hamari and Rowe [18] for measuring the flow in gamified learning environments and adapted the short survey described by Coller, Shernoff, and Strati [19] for assessment of flow, i.e., the idea was adapted from an empiric research utilizing experience sample method (ESM) approach. The environment is uses a PC, projector and Microsoft Kinect-sensor. The key idea is to provide an immersive and engaging learning experience for each individual pupil in a classroom. The working hypothesis for this study is that immersion based on sufficient challenge and bodily interaction triggers flow that helps pupils to perform well in exercising elementary mathematics.

In this paper, we first present the related work in this field, followed by a description of our application. We then present our two user studies and their results. We conclude by discussing the implications of our results to kinesthetic elementary mathematics and creating flow with gesture modality.

\section{Related Work}

The concept of flow originated from Csikszentmihalyi's [7] and [21] research on the psychology of optimal experience, or the study of happiness, fun and well-being. Optimal experience is defined as "a deep sense of enjoyment that is long cherished, [that] does not come through passive, receptive, relaxing times" [21]. The term flow is also used to represent this feeling of complete engagement and immersion in an activity. It is widely noted that during a state of flow, people lose track of time and are completely focused on the activity at hand, commonly referred to as 'being in the Zone' [20]. Chen [20] also describes Csikszentmihalyi's eight major components of flow as: (a) a challenging activity requiring skill, (b) a merging of action and awareness, (c) clear goals, (d) direct, immediate feedback, (e) concentration on the task at hand, (f) a sense of control, (g) a loss of self-consciousness, and (h) an altered sense of time. He also argued that being in a state of flow maximizes our performance in and pleasurable feelings from the activity. Although, being in the zone is more commonly associated with the arts and sports, it is in fact a feeling common to every individual, this feeling or state of optimum experiences, regardless of profession.

From Csikszentmihalyi's eight components of flow, it can be seen that various activities, especially with current day technologies such as video games, can support the state of flow. Games that are challenging and highly interactive have been found to promote a state of flow [7] and [8]. Games provide a challenging premise and it can be argued that the skills of players are developed further with gameplay. Furthermore, games provide realizable challenges with an appropriate level of feedback and reward to be engaging and interesting for the player, which are also essential conditions for flow. There can be various goals or levels of challenges in digital games such that players start from a basic skill level and build towards increasing complexity at their own controllable pace. As explained by McGonigal [8] "in a good computer or video game you're always playing on the very edge of your skill level". These interactive digital games also provide real time feedback to player's responses, thus "in computer video games, the interactive loop is satisfying tight" [8]. These attributes of digital games advocate a state of flow, where a player is completely absorbed in the game.

Furthermore, games that employ bodily interaction have been found to be highly immersive and engaging [1][2][3][4][5]. There are several known benefits of using bodily interactions in educational games; they allow even the more restless of students to be able concentrate for longer durations and improve classroom learning performance by introducing elements of playfulness and immersion [5]. In fact, recent studies in clinical psychology on children with Attentiondeficit/hyperactivity disorder (ADHD) strongly suggest that hyperactivity or gross motor activity can facilitate cognitive functioning [9]. ADHD is "a complex neurodevelopmental disorder which includes inappropriate levels of inattention, impulsivity, and hyperactivity" [9] which is usually presents itself as an undesirable aggressive behavioral characteristic. Yet, as shown by Saver et al. [9], children with ADHD perform better on memory related task when they are motorically active, findings that resonate with Pelham et al. [10]. Additionally, the learning from bodily interaction is 
also retained longer as seen after delayed tests of the learned material [2][11]. Overall, bodily interaction, by virtue of its cognitive potential and situational context, creates a state of flow.

Our study is based on digital educational games that employ bodily interaction techniques, which are highly immersive and provide an engaging learning environment, creating a state of flow. Our kinesthetic elementary mathematics application provides digital content for abstract mathematical concepts, in this case, of quantity and numerosity. Using similar metaphorical representations and bodily interaction, several mathematical games have been developed by researchers across the world. Lee, Liu and Zhang [4] focused more specifically on elementary school children, who have difficulty learning arithmetic, and designed and developed a space themed math game using a Microsoft Kinect. Isbister et al. [15] focused on math anxiety and the potential of using 'power poses' or gestures that increase confidence during interactions. There are also several independent teams from across the globe, for instance KinectMath, MathNUI [22] and Learning Math Using Gesture [16] working on introducing Kinect-based application within the classroom environment for teaching more complex mathematical concepts in higher education. Thus, bodily interactions are especially helpful in learning abstract metaphorical concepts [17].

\section{Application Design}

To validate the working hypothesis of bodily interaction increasing immersion and promoting flow, a test setting was staged. The test setting consists of a $2 \mathrm{~m} \mathrm{x} 3 \mathrm{~m}$ projection by a HD projector, a PC and Kinect-sensor, and an elementary mathematics application. The application consists of two different exercises with gesture interaction modality: a recognition game and a calculating game.

The recognition game requires pupils to connect a quantity to a number symbol as shown in Figure 1. After recognition or processing, the pupil should place a hand on the correct balloon, selecting it by pointing for a specified dwell time of three seconds, and then dragging the balloon to a bucket on the bottom right corner The calculating game includes the same colorful balloons as shown in Figure 1 , but the task is to add or subtract two numbers. One number and outcome are visible and the pupil has to catch a suitable balloon to match the answer. The application then provides feedback on whether it was the correct balloon/answer or not. Application counts points based on the number of correct answers.

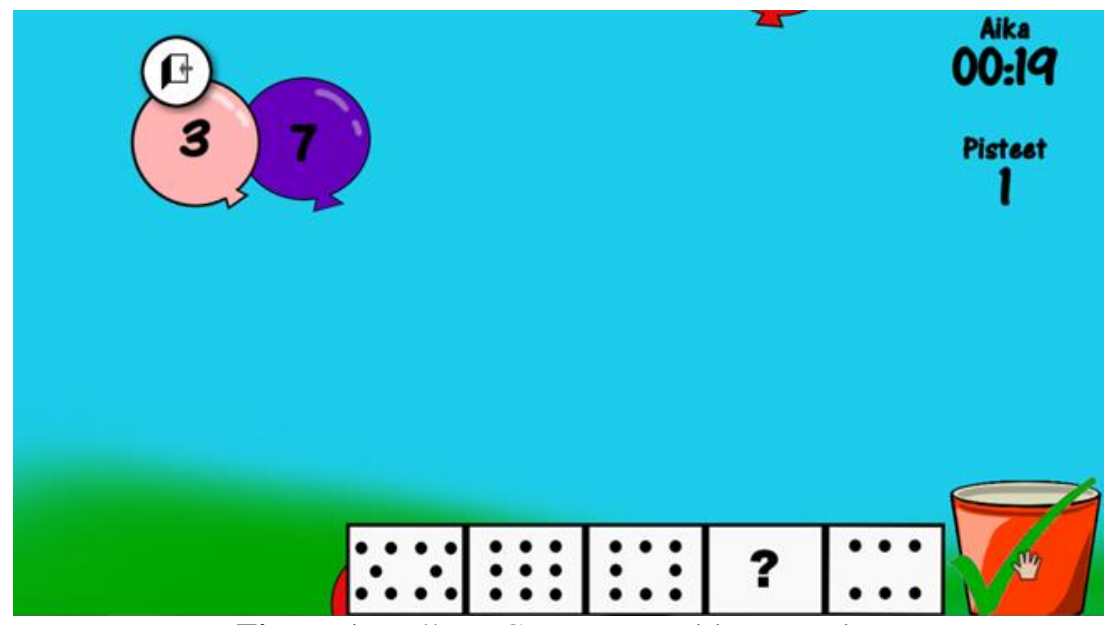

Figure 1: Balloon Game, recognition exercise

The Cubes game is has similar game logic as Tetris has. Falling cubes should be placed with suitable cube to get sum presented on the right-hand side of the screen as shown in the Figure 2. There are several options to place the cube, so pupil has to simultaneously do the adding and decision on which place to set the cube. If the moving cube falls to an incorrect place a new cube will appear. After placing a cube on a right cube, both cubes will disappear making more space to playing area. Game ends when any stack of cubes reaches the top of the playing area. 


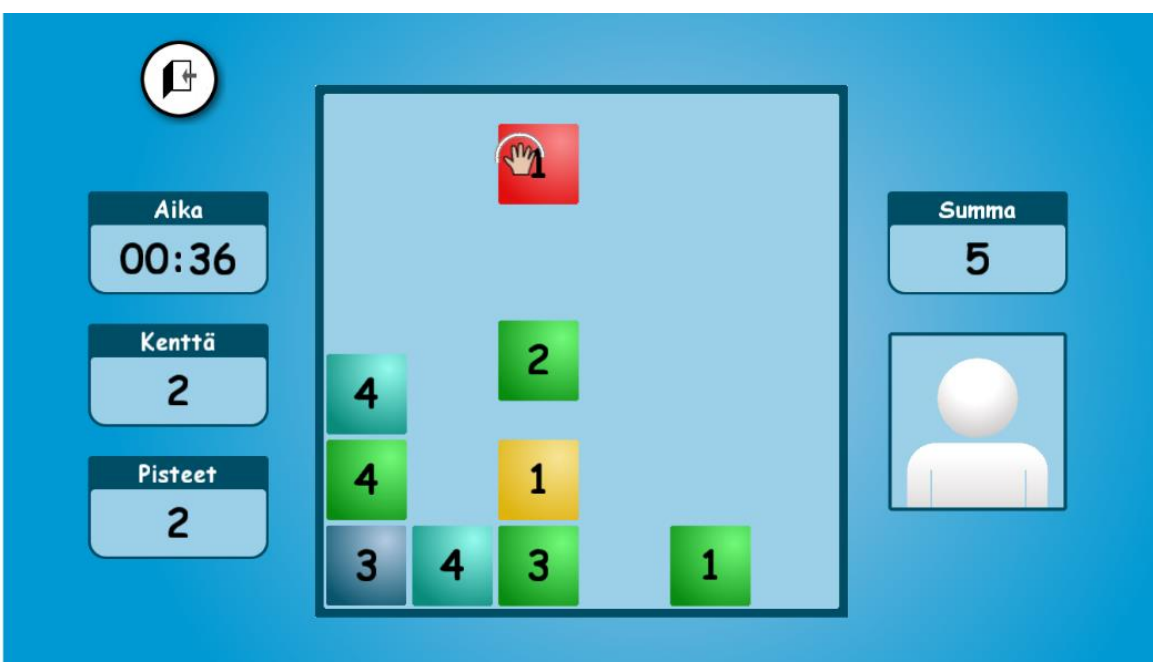

Figure 2: Cubes Game, adding exercise

The exercises in the application are based on first grade mathematics as the target group is first graders. Similar exercises can be found in mathematics books and digital exercises. The tasks, i.e. recognition of quantities and/or number or simple operations, are validated and widely in use in elementary schools. Moreover, as the application was designed for the assessment of effects of changing modality from written to bodily interaction, the tasks were kept simple as possible.

\section{Methods and Results}

The empirical part of the study consists of two evaluation rounds. The first round was to test and fine-tune the application, yet it also served as a preliminary proof of our working hypothesis. Results of the first round are more qualitative. The second round was set to test the working hypothesis and sessions with the application were conducted as unified as possible. Moreover, in both rounds respective teachers provided feedback on performance of the pupils. In the first round it was free form. In the second round it was structured, based on predefined questions. The methodology and outcomes of the first round are discussed first, and then of the second round.

\subsection{First Evaluation Round}

In the first round, the pupils played the game for 10-20 minutes without a preset target score. The unstructured sessions were more to evaluate first order user experience and usability of gesture modality in the context of elementary mathematics. The pupils played with the application for exercising and afterwards they were interviewed about their performance, immersion and concentration. Their respective teacher monitored the performance and gave feedback after each playing sequence. The teachers had six items to pay attention to and they assessed them by free form feedback. The findings were categorized and analyzed by the researchers as described in figure 2

\subsubsection{Participants}

The pupils were recruited from two different skills group. The first group was the target group, i.e. first grade pupils. The second group was pupils with special needs, whose skill level on mathematics was on the level of the first graders. The underlying assumption was that the flow stage would be different within each group. Moreover, it was assumed that the skill level would affect the overall experience with the game. As the pupils were selected by a convenience sampling, i.e. voluntarily by their teacher, the researchers had no recognition of the level of skills of an individual pupil. To meet this challenge the teacher assessed each pupil as described next.

The number of the participants in the first evaluation was 23. 20 of them were first grade pupils and three of them were pupils with special needs, but learning the same content with the first graders. In addition, two teachers participated and commented on the evaluation sequences. 


\subsubsection{Results of the first evaluation}

The first evaluation with the pupils was conducted in a classroom environment during spring 2013. The purpose of this evaluation was to test the functionalities of the elementary mathematics application with users, to evaluate the application by the teachers and to identify any difference with users with varying skills. Moreover, the evaluation also served the function of testing the overall research setting. The evaluation was based on two qualitative datasets. The first set was an assessment by researchers and it was about the general usability of the application. The second set was an assessment by the teachers and it was about how each pupil performed with the application.

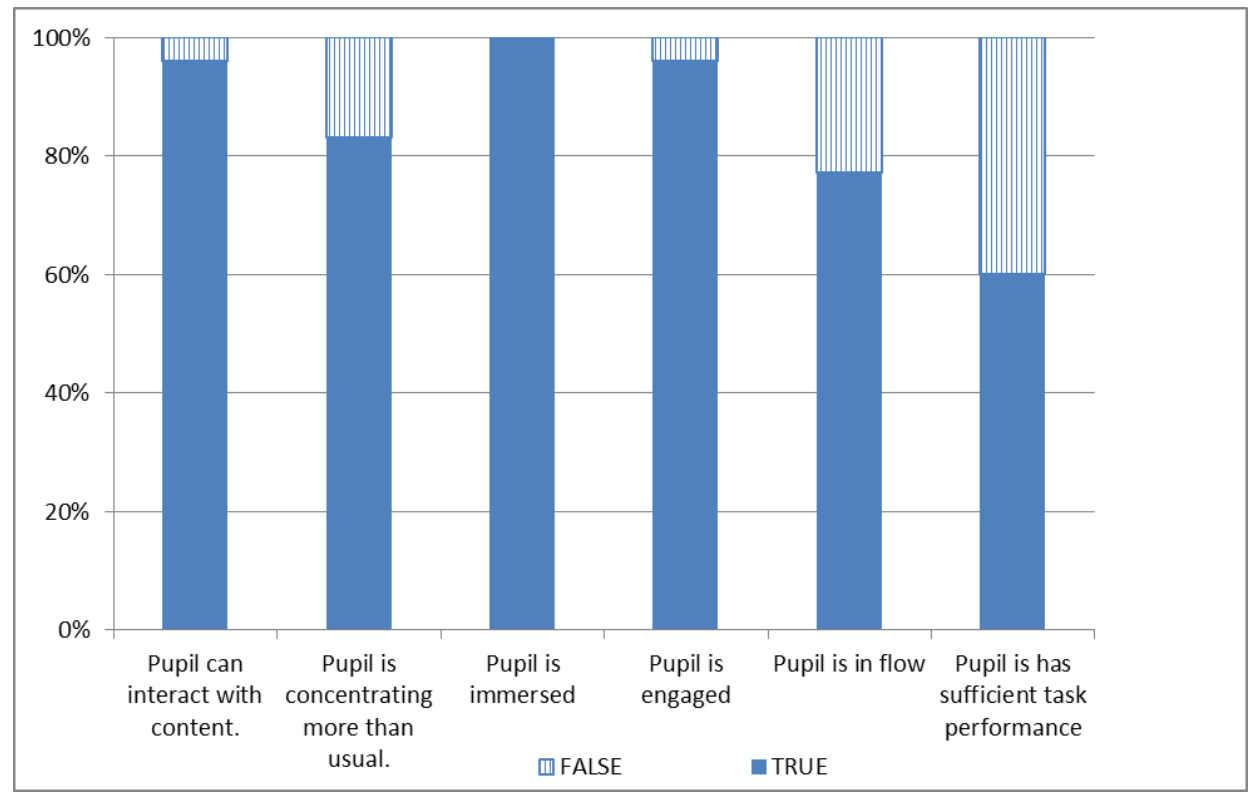

Figure 3: Percentage TRUE / FALSE response from teacher assessments

The key findings in the first evaluation are related to interaction, immersion and engagement as described in Figure 3. According to the teachers, all pupils gained from the bodily interaction and large projection. Attention was drawn to changed concentration of the pupils, as standing and steering with gestures increased their concentration and attention, especially for those pupils with short attention span. They concentrated more than what they would in a traditional classroom setting. The immersion of the application was also found useful, as exercising with it was assessed more holistic. Due to the attractiveness of the application, the engagement in usage was also evaluated higher than usual. The teachers did not find any significant differences between first graders and pupils with special needs. The first evaluation of the interaction revealed some shortcomings with gesture interaction, for example, exhaustion during long exercising and inaccurateness in boundary areas of the projection. $40 \%$ of pupils did not perform as they were expected,. The findings of the first evaluation were taken into account when the application was iterated and developed further. The most important changes were to limit and fine tune the gestures; that is, decreasing the area where hand for grabbing was supposed to be and replacing grabbing with waiting and setting idle time to 3 seconds.

In conclusion, the main findings of the first evaluations are related to immersion, engagement and flow. The researches evaluated engagement and concentration on the game and the teachers evaluated concentration, immersion and flow. As expected, pupils with typically developed skills and with special needs were immersed, concentrating and in flow. Moreover, the evaluations revealed that gesture modality is affordable, easy to access, and an enjoyable way to interact with digital content. The poor task performance was due to shortcomings in the user interface and wrong assumptions when the user interface was developed.

\subsection{Second Evaluation Round}

In the second round the pupils were asked to play two rounds of the mathematics exercise game that was iterated version with enhanced user interface and content. Each pupil played the game until 
reaching a total score of 20 or spending the allocated time of two minutes for both games. The average duration of playing was 4 minutes for the calculating game, and 3 minute 15 second for the recognition game.

During the second evaluation, each pupil assessed the experience of exercising with the application using the Experience Sampling Method (ESM) questions described by Coller, Shernoff, and Strati [19]. The ESM method embeds short survey questions into the playing of a game, at a variety of key moments, in order to administer flow measurement items that discern how much the activity feels like work and play to the participant, and examine levels of enjoyment, challenge, and interest at each designated point in the game. In this case, experience was evaluated after the exercise session since the duration of each session was short and to avoid additional distraction to performance. In addition, ESM was not utilized in an orthodox manner yet the key components of playing experience were caught even with this simplified way. The limitation in full scale utilization of ESM was the duration of the playing sequences and the exercise setting. The pupils did not have access to the application outside of the classroom or school hours, thus the experiment was limited to two playing sequences.

Table 1 shows the questions to the pupils and each question was answered with three options: positive, neutral and negative. The number of options was decreased from typical 7 or 5 point scale to 3 points because of the target group being children of age 7 to 13 years. In the survey form there was an emoticon that related to each option, i.e. $:-$ for positive, $:-$ for neutral, and $: \theta$ for negative. Such simplification of the scale is supposed to help pupils easily describe the experienced emotion on each item asked. The use of emoticons is justified, since not all attendees could read. In the test, the supervisor read the questions and the pupil chose a respective option.

Table 1: ESM questions

\begin{tabular}{c|c|c|c}
\hline Question & $\begin{array}{c}\oplus ; \\
\text { Positive }\end{array}$ & $\begin{array}{c}* \\
\text { Neutral }\end{array}$ & $\begin{array}{c}: \\
\text { Negative }\end{array}$ \\
\hline How interesting is it? & & & \\
\hline How much are you enjoying what you are doing? & & & \\
\hline How hard are you concentrating? & & & \\
\hline Is it challenging? & & & \\
\hline How skilled are you at the game? & & & \\
\hline
\end{tabular}

In addition to the user experience, teachers were asked to assess pupils' performance while playing. Teachers were asked four questions about the performance and performance was evaluated on a 5point scale. The question were: 1) Pupil is concentrating, 2) Pupil outperforms, 3) Pupil is capable of sufficient actions, and 4) Pupil is immersed. The teacher assessment was utilized in the second evaluation in 2014 and the respective teacher assessed the performance of each pupil in order to see expected performance level. In the first round evaluation teacher assessed the performance qualitatively.

\subsubsection{Participants}

The second evaluation was conducted as a test setting in order to maintain the commensurability of the results. In the test there were three different user groups: first graders $(n=17)$ with average variation of skills of number area for 0 to 20 , second graders $(n=18)$ with all mastering the number area, and pupils with special needs $(n=9)$ with some difficulties with the number area or operations. The total number of participants was 44 . Grouping was based on the working hypothesis that the target group (i.e., first graders) and pupils with difficulties are expected to experience flow with the application, yet for those who are already familiar with the content flow is hindered by frustration or trivialness. In addition to pupil participants, four teachers evaluated performance of the pupils.

\subsubsection{Results of the second evaluation}

The second evaluation was conducted in spring 2014 with more extensive content, better graphics and sound, and improved interaction, mainly reducing interaction fatigue by designing better projection boundaries, as explained in the results of the first evaluation. The research was set in a separate learning environment with suitable equipment instead of a classroom. The location does not 
matter per se as the designated space had more advantages than a classroom, such as, better projection space and lighting, favorable acoustics and more space for playful experience. The evaluation aimed to study how immersive bodily interaction promotes flow, i.e. how large projection with gesture interaction differs from traditional classroom exercise settings. However, with studies that employ novel interaction techniques it is difficult to eliminate the influence of the novelty of interaction on the participants' behavior. Therefore, this is also a limitation in our study and its results.

The second experiment was conducted to test the working hypothesis and pupils were grouped as described earlier. The skills level was set as an independent variable and the most evident findings are in accordance to it. In addition to direct user experience feedback, each pupil's performance was assessed by their respective teacher. This was a controlled setting, since the researchers had no knowledge of the skill level, which was set as independent variable. Moreover, issues in the performance of a pupil was unknown and therefore teacher insight was needed. In addition to experience and performance evaluation, pupils and teacher could provide free form feedback on the application.

Pupil responses, as Pac-man pie charts, are shown in 4. All first graders found the game interesting, but only half of the second graders gave the same evaluation. Overall, most of the special pupils found game interesting. The responses to with enjoyment was the similar. The children who did not find the game interesting or enjoyable also evaluated their level of concentration lower. Roughly 30 $\%$ of pupils, of all groups, agreed that the game required concentration. Most of them expressed that gesture interaction caused difficulties and required focused attention. Those who judged that playing the game was not challenging rated themselves also as skilled players. The general result of the pupils is that, those who are skilled enough and have some challenges with the content are immersed, concentrating and in flow to an extent. Moreover, those who had a negative experience towards the game were mostly second graders or pupils who faced serious difficulties with the content.

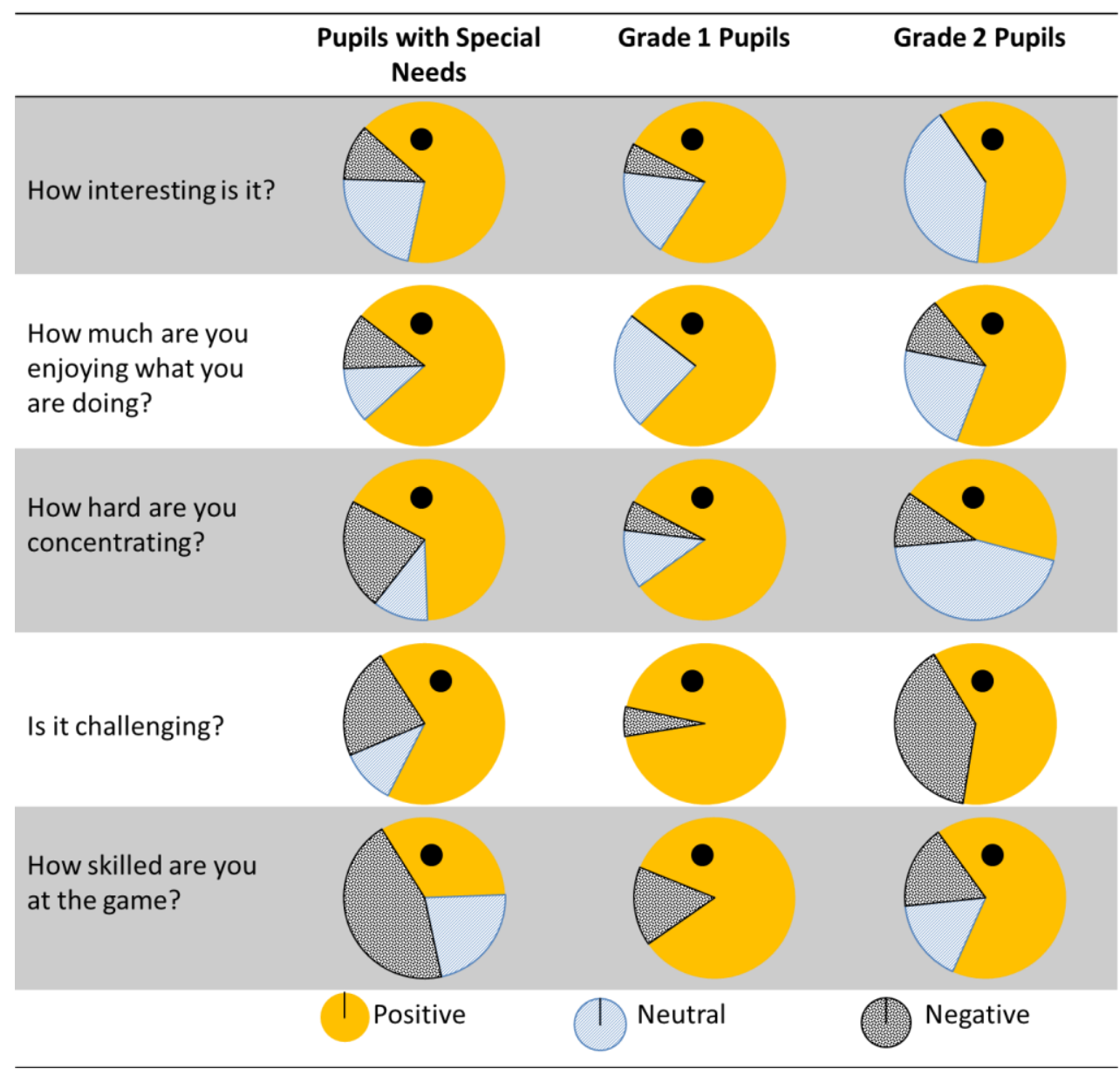

Figure 4: Pupil responses shown in Pac-Man Pie charts

The pupils were mostly positive towards the game and the assessment by the teachers supported this finding. In the survey, the teachers assessed concentration level quite high and in open ended answers 
they expressed that the game is well suitable for those who are still learning the content. The teachers stated that for some second graders, the game might be too easy and therefore caused the frustration, this explains the slightly lower averages in teacher assessments for second graders with respect to first graders, as shown in Figure 5. For first graders, teachers assessed high concentration and very good or excellent performance. Some pupils with special needs did have difficulties with gesture interaction, which affected the teacher assessments of their performance (Figure, question B). However, some of the gestures difficulties were due to varying physical capabilities and interests of the pupils with special needs. It would be interesting to study and design a gesture vocabulary that is comfortable and exciting for pupils with special needs to further enhance their experiences. On five point scale, teachers assessed overall mean concentration to 4.6 ( $\mathrm{SD}=0.78)$, mean outperformance to $3.7(\mathrm{SD}=1.37)$, overall performance to $4.4(\mathrm{SD}=1.04)$, and immersion to 4.5 ( $\mathrm{SD}=0.87)$. In general, the teachers' assessment confirmed our hypothesis; how immersion and sufficient challenge affect the performance or how pupils will outperform.

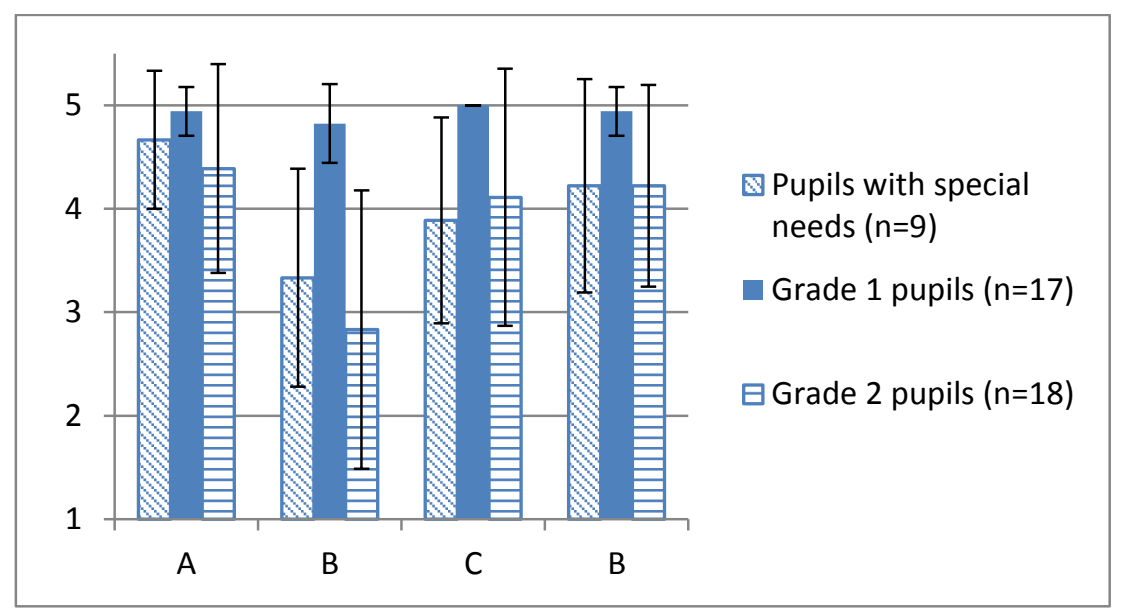

Figure 5: Grade-wise teacher assessments to questions (A) pupil is concentrating, (B) pupil outperforms, (C) pupil is capable of actions, and (D) pupil is immersed.

Thus, the answers about concentration, skills and challenge support our working hypothesis. All pupils from groups one and three assessed their concentration positive. Over $70 \%$ found the application challenging. Less than $15 \%$ thought that they lacked the required skills. Comparing that to teachers' assessment, a clear distinction for group two could be noticed, highlighting the most important issues about immersion and flow the teachers assessed that high concentration and immersion caused excellent performance and pupils to outperform.

Moreover, according to the teachers, most of the first graders did perform better with the application that in a normal classroom settings. For grade 2 pupils who did not perform well or did not concentrate, the most probable reason was the trivialness of the content to players, i.e. they were too familiar with the topic and it caused disinterest. The teachers did not make distinctions between first graders and those with special needs, as both these two groups gained in this setting. The effect on the second graders was considered neutral or even negative by the teachers.

In the open ended answers the teachers emphasized and confirmed the value of bodily interaction to those who have difficulties to concentrate in normal situations, e.g. those with special needs or pupils with learning challenges. Some of the pupils were acknowledged as kinesthetic learners and for them tangible interactions provided a more conducive learning environment. According to the teachers, the target group, i.e. pupils still learning the content, were benefitted from using the application. Gesture modality was found useful, yet it has posed some restrictions on non-accustomed users. The teachers assessed that the application promotes learning and they also found it very useful for teaching this topic.

\section{Conclusions and discussion}

In conclusion, it can be stated that our findings, from the two evaluations of gesture interaction applications for elementary mathematics, are encouraging. As the empirical work with school children is still mostly experimental, no strong conclusions about learning outcomes or comparisons between user groups can be made. However, as a first set of field studies with the application, the 
working hypothesis was confirmed, i.e., immersion and engagement triggered by bodily interaction enable concentration and flow. Even in a study of this extent, the flow hypothesis was verified by the subjective measurements and assessment by the teachers. The results of this study are still suggestive due to limited number of participants and a non-controlled test setting.

The tested research setting requires some modifications, yet it serves the function sufficiently. The major difficulty with such settings is adjusting subjective assessment by the user. The two tasks are quite different, because recognition is substantially different from calculation and performance on different types of tasks were not evaluated separately. The need for practical measurement, i.e. minimizing the burden of measuring, is somewhat contradictory to the need for thorough assessment. The modified method used for acquiring user data is a kind of a compromise between those two issues, yet due to its simplified scale, the same level of validity cannot be reached as a full scale ESM measurement setting. However, the reliability of the results can be considered high and it has more weight when assessing the results. Moreover, the reliability of the results is enhanced by the auxiliary data set provided by the teachers. The teachers also confirmed the conclusions drawn from the pupil data.

Our conclusions resonate with Csikszentmihalyi's [7] and [12] eight major components of flow. As our results show, pupils who found the activity challenging for their individual's skill level but not impossible to complete, achieved a state of flow. As also stated by McGonigal [8], a balance between the challenge and the player skill levels should be met. Furthermore, pupils who had a negative attitude towards the application were those who faced difficulties with the content. For them the game was too challenging, and not meeting the challenge vs skills balance. The game had clear goals and, direct and immediate feedback for the participants. Moreover, it was noted that pupils concentrated on performing the correct gesture for interaction, by standing intently and with focused steering of gestures. This focus led to a sense of control in interaction with the application, merging the action and awareness towards a more holistic experience.

Chen [20] also described flow as being in the zone. This was emphasized in our evaluation by the teachers who noted that bodily interaction promoted immersion and engagement enabling concentration and flow. Furthermore, the teachers noted that bodily interaction promoted a high level of concentration and immersion resulting in excellent performance from the pupils, as also stated by Chen [20]. Previous research has already shown that bodily interaction in games is highly immersive and engaging [1][2][3], [4][5], which the teachers in our evaluation also expressed. Moreover, the teachers identified a connection between enjoyment and immersion or concentration, which resonates with Chen's [20] statement of being in the zone; that is, both performance and pleasure are optimum in a state of flow.

Additionally, the teachers emphasized that pupils with difficulties in concentrating in their classroom sessions were able to pay more attention to the application because it employed bodily interaction. This finding corresponds with previous studies with children with Attention-deficit/hyperactivity disorder (ADHD); that is, gross motor activity facilitates cognitive functioning [9] and increase concentration and classroom learning performance [5].

\section{Acknowledgements}

The platform utilized in the realization of this study was originally the outcome of a Finnish Technology Agency funded project "Active Learning Spaces" and two coding projects conducted in University of Tampere, Finland. We acknowledge inputs of Ms. Maarit Laitinen, Dr. Sari Yrjänäinen and the coding teams for their work on gesture-based learning environment for elementary mathematics.

The research and field work was conducted with support of Finnish Technology agency funded project "FUN: Finnish - US Network" http://www.uta.fi/sis/trim/groups/rime/projects/fun.html

\section{References}

[1] Abrahamson, D., \& Trninic, D. (2011, June). Toward an embodied-interaction design framework for mathematical concepts. In Proceedings of the 10th International Conference on Interaction Design and Children (pp. 1-10). ACM. http://dx.doi.org/10.1145/1999030.1999031 
[2] Chang, C. Y., Chien, Y. T., Chiang, C. Y., Lin, M. C., \& Lai, H. C. (2013). Embodying gesturebased multimedia to improve learning. British Journal of Educational Technology, 44(1), E5E9. http://dx.doi.org/10.1111/j.1467-8535.2012.01311.x

[3] Dede, C. (2009). Immersive interfaces for engagement and learning. Science, 323(5910), 66-69. http://dx.doi.org/10.1126/science.1167311

[4] Lee, E., Liu, X., \& Zhang, X. (2012). Xdigit: An arithmetic kinect game to enhance math learning experiences. Retrieved February, 14, 2013.

[5] Lee, W. J., Huang, C. W., Wu, C. J., Huang, S. T., \& Chen, G. D. (2012, July). The Effects of Using Embodied Interactions to Improve Learning Performance. Advanced Learning Technologies (ICALT), (pp. 557-559). IEEE.

[6] Lindgren, R., \& Johnson-Glenberg, M. (2013). Emboldened by Embodiment Six Precepts for Research on Embodied Learning and Mixed Reality. Educational Researcher, 42(8), 445-452. http://dx.doi.org/10.3102/0013189X13511661

[7] Nakamura, J., \& Csikszentmihalyi, M. (2002). The concept of flow. Handbook of positive psychology, 89-105.

[8] McGonigal, J. (2011). Reality is broken. Jonathan Cape, London.

[9] Sarver, D. E., Rapport, M. D., Kofler, M. J., Raiker, J. S., \& Friedman, L. M. (2015). Hyperactivity in Attention-Deficit/Hyperactivity Disorder (ADHD): Impairing Deficit or Compensatory Behavior?. Journal of abnormal child psychology, 1-14. http://dx.doi.org/10.1007/s10802-015-0011-1

[10] Pelham, W. E., Jr., Waschbusch, D. A., Hoza, B., Gnagy, E. M., \& Carter, R. L. (2011). Music and video as distractors for boys with ADHD in the classroom: comparison with controls, individual differences, and medication effects. Journal of Abnormal Child Psychology, 39, 1085-1098. http://dx.doi.org/10.1007/s10802-011-9529-z

[11] Chao, K. J., Huang, H. W., Fang, W. C., \& Chen, N. S. (2013). Embodied play to learn: Exploring Kinect-facilitated memory performance. British Journal of Educational Technology, 44(5), E151-E155. http://dx.doi.org/10.1111/bjet.12018

[12] Lakoff, G., \& Núñez, R. E. (2000). Where mathematics comes from: How the embodied mind brings mathematics into being. Basic books.

[13] Roth, W. M., \& Thom, J. S. (2009). Bodily experience and mathematical conceptions: From classical views to a phenomenological reconceptualization.Educational Studies in Mathematics, 70(2), 175-189. http://dx.doi.org/10.1007/s10649-008-9138-0

[14] Moeller, K., Fischer, U., Link, T., Wasner, M., Huber, S., Cress, U., \& Nuerk, H. C. (2012). Learning and development of embodied numerosity. Cognitive processing, 13(1), 271-274. http://dx.doi.org/10.1007/s10339-012-0457-9

[15] Isbister, K., Karlesky, M., Frye, J., \& Rao, R. (2012, May). Scoop!: a movement-based math game designed to reduce math anxiety. In CHI'12 Extended Abstracts on Human Factors in Computing Systems (pp. 1075-1078). ACM.

[16] Thakkar, V., Shah, A., Thakkar, M., Joshi, A., \& Mendjoge, N. (2012, July). Learning Math Using Gesture. In Education and e-Learning Innovations (ICEELI), 2012 International Conference on (pp. 1-3). IEEE. http://dx.doi.org/10.1109/iceeli.2012.6360617

[17] Hashagen, A., Büching, C., \& Schelhowe, H. (2009, June). Learning abstract concepts through bodily engagement: a comparative, qualitative study. In Proceedings of the 8th International Conference on Interaction Design and Children (pp. 234-237). ACM. http://dx.doi.org/10.1145/1551788.1551839

[18] Shernoff, D. Hamari, J., Rowe, E. (2014) Measuring Flow in Educational Games and Gamified Learning Environments. World Conference on Educational Multimedia, Tampere, Finland

[19] Coller, B. D., Shernoff, D. J., \& Strati, A.D. (2011) Measuring Engagement as Students Learn Dynamic Systems and Control with a Video Game." Advances in Engineering Education 2.3

[20] Chen, J. (2007). Flow in games (and everything else). Communications of the ACM, 50(4), 3134. http://dx.doi.org/10.1145/1232743.1232769

[21] Csikszentmihalyi, M., \& Csikzentmihaly, M. (1991). Flow: The psychology of optimal experience (Vol. 41). New York: HarperPerennial.

[22] Echeverría, M. A. M., Santana-Mancilla, P. C., Carrillo, H. F. Q., \& Enciso, E. A. F. (2013). Natural user interfaces to teach math on higher education. Procedia-Social and Behavioral Sciences, 106, 1883-1889. http://dx.doi.org/10.1016/j.sbspro.2013.12.214 